\title{
Matching Ophthalmology amidst Coronavirus Disease 2019 (COVID-19): Lessons That Went Viral
}

\author{
Harris Ahmed, DO, MPH ${ }^{1}$ Janice C. Law, MD² David Felsted, DO ${ }^{3}$ Sheena Song, $\mathrm{DO}^{4}$ \\ Tamzid Rahman, DO, MPH ${ }^{5}$ Jennifer L. Lindsey, MD $^{2}$ Rachel Spurrier, DO ${ }^{3}$ \\ Andrew J. Hendershot, $\mathrm{MD}^{6}$ Bilala Ahmed, $\mathrm{BS}^{7}$ Nisha Chadha, $\mathrm{MD}^{8}$ \\ ${ }^{1}$ Department of Ophthalmology, Loma Linda University, Loma Linda, \\ California \\ ${ }^{2}$ Department of Ophthalmology and Visual Sciences, Vanderbilt \\ University Medical Center, Nashville, Tennessee \\ ${ }^{3}$ Department of Ophthalmology, Medical College of Georgia, \\ Augusta, Georgia \\ ${ }^{4}$ Department of Ophthalmology, St. John's Episcopal Hospital, Far \\ Rockaway, New York \\ ${ }^{5}$ Department of Ophthalmology, Larkin Community Hospital, South \\ Miami, Florida \\ ${ }^{6}$ Department of Ophthalmology and Vision Sciences, The Ohio State \\ University Wexner Medical Center, Columbus, Ohio \\ ${ }^{7}$ University of Toledo College of Medicine, Toledo, Ohio \\ ${ }^{8}$ Department of Ophthalmology, Icahn School of Medicine at Mount \\ Sinai/New York Eye and Ear, Eye and Vision Research Institute, New \\ York, New York \\ J Acad Ophthalmol 2020;12:e200-e204.
}

The coronavirus disease 2019 (COVID-19) pandemic has changed medical education and the residency match process for both medical students and residency programs, with ophthalmology being no exception. Medical students now face cancelled away rotations, virtual interviews, limited interactions with programs, and overall apprehension about changes to the San Francisco Match timeline.

Matching into ophthalmology continues to be exquisitely competitive. Over the last three years, the match rate for applicants has been between 75 and $78 \%$. Data gathered from the preceding 10 years demonstrate that the mean USMLE (United States Medical Licensing Examination) step 1 score of matched students has increased by nearly 10 points to a matched mean of 245 in 2020. Furthermore, to secure a position, students are now applying on average to 77 programs, about a $50 \%$ increase in applications per applicant compared with a decade ago. ${ }^{1}$ Beyond USMLE scores, letters of recommendation, research, and away rotations have been strongly associated with a successful match. ${ }^{2}$ Additionally, the presence of a home ophthalmology residency program is also strongly associated with matching, with data revealing that attending a medical school with a residency program increases an applicant's likelihood of matching by 1.4 -fold. ${ }^{3}$ Previous research has shown that the majority of applicants

Address for correspondence Harris Ahmed, DO, MPH, Department of Ophthalmology, Loma Linda University, 11370 Anderson Street \#1800, Loma Linda, CA, 92354 (e-mail: hahmed@llu.edu). match at a residency program within the same geographic region as their medical school. ${ }^{3}$ According to these data and many documented experiences, ${ }^{4}$ students without home residency programs or ophthalmology departments are at an incredible disadvantage. Currently, only $53 \%$ of U.S. medical schools are associated with an ophthalmology residency program and $58 \%$ have an ophthalmology department ( - Table 1). Thus, many medical students have limited access to ophthalmologists, research, and networking opportunities. Furthermore, seeking opportunities from departments outside one's medical school often comes with logistical issues, time constraints, and financial burden.

Although both applicants and residency programs alike are struggling to navigate this cycle, students without home programs will disproportionately be affected. In an effort to mitigate the impact of COVID-19 on match applicants this year, six of us, ranging in training from recently matched MS$4 \mathrm{~s}$ to PGY-4s, collaborated to host a webinar to guide students during this unprecedented situation. Each panelist came from a school without an ophthalmology department and felt a strong duty to give back to students in the form of mentorship, information, and encouragement.

We will share lessons, experiences, and feedback we have received from our webinar in the hope that it may inform
Copyright $\odot 2020$ by Thieme Medical Publishers, Inc., 333 Seventh Avenue, New York, NY 10001, USA. Tel: +1(212) 760-0888.
License terms 10.1055/s-0040-1716412. ISSN 2475-4757.

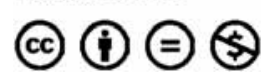


Table 1 Comparison of medical schools with and without ophthalmology departments and/or home ophthalmology residency programs

\begin{tabular}{|l|l|l|}
\hline & No. of medical schools & Percentage of medical schools \\
\hline $\begin{array}{l}\text { Combined AAMC and AACOM list: total number of } \\
\text { medical schools (allopathic and osteopathic) }\end{array}$ & 194 & 100 \\
\hline ACGME list: medical schools with home residency program ${ }^{\text {b }}$ & 103 & 53 \\
\hline $\begin{array}{l}\text { AUPO members list: medical schools with home } \\
\text { ophthalmology department }\end{array}$ & 113 & 58 \\
\hline $\begin{array}{l}\text { Combined AAMC and AACOM list: medical schools without } \\
\text { any ophthalmology department or residency program }\end{array}$ & 81 & 42 \\
\hline
\end{tabular}

Abbreviations: AACOM, American Association of Colleges of Osteopathic Medicine; AAMC, Association of American Medical Colleges; ACGME, Accreditation Council for Graduate Medical Education; AUPO, Association of University Professors of Ophthalmology.

${ }^{\text {a Per combined AAMC }}{ }^{10}$ and AACOM ${ }^{11}$ medical school roster list for 2019-2020 academic year in 50 U.S. states excluding military programs.

'Per ACGME 2019-2020 list of ophthalmology residency programs. ${ }^{12}$

'Per AUPO 2020 member institution list. ${ }^{13}$

others on the needs of applicants, the power of social media as a tool for mobilization and collaboration, innovative approaches to mentorship, and the unique demands that exist for this cycle.

Lesson 1

Medical education, mentorship, and information can be delivered, with no cost, to tens of thousands through social media content engagement by key influencers. Social media does not follow the same hierarchy as academic medicine. A medical student can often have more influence and reach than a senior attending or program director. We will outline exactly how our webinar went viral.

Lesson 2

COVID-19 has complicated this cycle for both students and programs, but consequences of COVID-19 disproportionately affect students without home departments or programs. The struggles these students face existed before the pandemic, are magnified due to the pandemic, and will likely remain afterward. We will outline how students without home departments or residency programs are perpetually disadvantaged in a way that transcends this pandemic.

Lesson 3

There is a great need and potential in creating a mentorship program for students without home programs and departments to provide mentorship, networking, and research opportunities remotely. Social media provides a premier platform to recruit mentors and reach students. We will outline how we developed an ophthalmology mentorship list and how others are developing an ophthalmology mentorship program.

\section{Reach and Impact}

What was initially meant to be an intimate session between six residents and a handful of students transformed into an internationally attended webinar with nearly 200 attendees, featuring collaborations with program directors and faculty from across the country. Organizers of the webinar were $\mathrm{Dr}$ Harris Ahmed of Loma Linda University, Dr David Felsted and Dr Rachel Spurrier of Medical College of Georgia, Dr Sheena Song of St. John's Episcopal, Dr Tamzid Rahman of Larkin Community, and Dr Ryan Machiele of University of North Carolina at Chapel
Hill. Attendees from the San Francisco Match (SFmatch), American Academy of Ophthalmology (AAO), American University Professors of Ophthalmology (AUPO), and American Osteopathic College of Ophthalmology and Otolaryngology - Head and Neck Surgery (AOCOO-HNS) were in attendance. Attendees came from nine countries and most of the United States, including many states without ophthalmology residency programs, such as Idaho, New Mexico, North Dakota, and Nevada. Other countries represented were Sri Lanka, Singapore, India, Nepal, Morocco, England, Canada, and Ecuador.

The original post advertising our webinar was posted on Twitter through the @drharrislakers Twitter account. The Tweet featured an advertisement banner that contained the purpose of the webinar and meeting information. Within 4 days, our webinar banner exceeded 16,000 views from one account alone (@drharrislakers) on Twitter. We subsequently shared the banner on Instagram. On Instagram, our stories and posts aggregated more than 30,000 impressions, meaning that 30,000 individuals saw our content, from just three accounts (@drharrislakers, @felmology, @sheena.eye.am). Our three Instagram accounts have a combined total of 5,000 followers, yet our posts reached six times our follower count, indicating that our posts and stories were widely disseminated and shared beyond our social media circles.

Medical students began flooding our direct messages requesting the webinar link, sharing excitement and gratefulness. How did we go viral? During the pandemic, we have seen a significant increase in the sharing of ophthalmic information through social media ${ }^{5}$ and noticed a dramatic increase in views/impressions, retweets, and comments once we had engagement and support from ophthalmologists with a large social media presence, such as Dr Andrea Tooley (@DrAndreaTooley), Dr Uswoma Abugo (@MentorMeMD), and Dr Janice Law (@janicelawmd). These high-profile influencers were not sought out nor did we request them to post our webinar on their accounts. Instead, they become aware of the webinar by word of mouth, retweets, and through students directly tagging them in tweets mentioning the webinar. The first lesson we learned is that information can be delivered to "masses" of interest through identifying key influencers. Within ophthalmology, this means identifying individuals 
with a large following and high engagement (likes and comments on posts and Tweets) and requesting them to support and spread information. Interestingly, social media does not follow the same hierarchy as academic medicine: a medical student can have more influence than a senior attending or program director. In our case, one medical student from the University of Toledo, Bilal Ahmed (@bilal_1712), was critical in identifying and tagging key influencers on Twitter to promote our webinar. Bilal contributed significantly to the virality of the webinar as the programs, faculty, and influencers he tagged in Tweets related to our webinar made the tagged individuals more likely to see our advertisement banner.

What happened next was even more surprising. No longer was it just students reaching out, but we received messages from program directors, faculty, and fellows from across the nation, all offering unwavering support and volunteering to mentor students without home programs and departments. We were also messaged by representatives from the SF Match and AOCOO-HNS. SF Match leadership requested us to provide a list of FAQ based on questions and concerns of attendees, and the AOCOO-HNS formally promoted our webinar. In addition to these organizations, we received encouragement from individuals from both AAO and AUPO leadership.

\section{Webinar}

During the webinar, we were joined by residency directors from Iowa, Ohio State, and Vanderbilt, as well as directors of medical student education and other faculty from Northwestern, New York Eye and Ear Infirmary of Mount Sinai, Vanderbilt, and Weill Cornell. Students shared concerns that they would not be able to effectively express interest in programs, lacked access to faculty who could advocate for them and write letters of recommendation, and did not have local research opportunities to participate in. We quickly discovered that many of the struggles that students were encountering existed before COVID-19, are currently being amplified due to COVID19 , and will likely continue after the pandemic is over. Fortunately, the webinar increased awareness of the challenges faced by this group of applicants. In response, many programs offered to grant exceptions and accommodate local students without home programs for away rotations, if possible. However, we believe that unless changes are made, talented students without home programs will continue to be overlooked due to factors largely out of their control.

While panelists were able to provide much needed information and encouragement, we were unable to resolve many of the other needs of applicants. To mitigate these concerns, we developed a mentor list for students without home programs or departments to be able to contact. We issued a call for mentors through Twitter, and we were again floored by responses. Our mentor list consists of faculty from across the country, including faculty from Bascom Palmer, Iowa, UCLA, Ohio State, Vanderbilt, Case Western, and Weill Cornell. These faculty members have committed to providing students with mentorship, advocacy, and research projects during this cycle.

One brief example of our success involves a medical student in Maine. After joining the webinar, the student reached out to one of our panelists on Instagram (Dr. Tamzid Rahman, @tzidd) to inquire about potential research and clinical experience. He was able to connect her directly with the administration at the Maine Eye Center, and she is now establishing research and clinical experience. This brings us to a third lesson: that there is great need and potential in creating a mentorship program whereby students without home programs and departments can be partnered with willing mentors to provide mentorship, networking, and research opportunities remotely. Such a program or database would address many of the challenges students face from smaller allopathic and osteopathic schools as well as provide research projects for willing and eager students. Luckily, one such virtual mentoring program is being developed by $\mathrm{Dr}$ Andrea Tooley, Dr Janice Law, and Dr Uswoma Abugo. ${ }^{6}$

\section{Future Directions}

Our experience offers an example of how social media can be leveraged to overcome some of the barriers to the residency application process in the COVID era and beyond. Specific to the advancement of medical education, webinars can be used for information dissemination, for idea exchange, and to foster collaboration and virtual networking. This experience may be applicable to students seeking residencies in other fields of medicine and to training programs alike, providing a platform to share information about their departments. Since our webinar, the AUPO conducted a webinar to help guide medical students applying to ophthalmology, which was also well attended and appreciated. Additionally, we have committed to a second webinar to discuss application strategy with applicants. We will be tracking outcomes of students who attended our webinar through surveys to explore the strengths, weaknesses, and overall effectiveness of using webinars and social media to disseminate knowledge, provide mentorship, and, ultimately, match into ophthalmology.

Many of our attendees were upperclassmen (MS-3 and MS-4) but we did have several MS-1 and MS-2 students in attendance. This brings up a larger discussion point, that is, students may benefit from earlier exposure to ophthalmology and mentorship. There has been a trend in previous decades of decreasing medical student exposure to ophthalmology in clinical years. ${ }^{7}$ Innovative approaches such as the Director of Medical Student Education (DMSE) position has been established to reverse this declining trend of ophthalmology exposure. The DMSE is an advocate for ophthalmology within medical schools and advocates for increased exposure to ophthalmology. Early research suggests that medical schools with DMSEs have higher rates of both required and extracurricular exposure to ophthalmology, as well as higher rates of faculty engagement with medical students. ${ }^{8}$ Social media and webinars may be mechanisms to enhance the impact and reach of DMSEs.

Social media has provided a radical transformation of access to information. Currently, students heavily rely on Web sites such as Reddit, Student Doctor Network, and the annual Ophthalmology applicant Google Excel sheet to obtain information and guidance on how to match into ophthalmology. The increasing popularity and engagement on 
social media has broken down traditional geographic and professional barriers, allowing students to directly connect with faculty physicians and potential mentors. Attendance at our webinar and subsequent follow-up by students with panelists reveal the immense need for mentorship and information in ophthalmology. Mentors have shown that they are eager to reach out to help students without home programs or departments. Perhaps, COVID-19 and the subsequent downturn in ophthalmology clinical volume created more time for academicians to participate in such mentoring initiatives, and we hope this trend is sustained. The benefits of mentorship are bidirectional, also providing programs with an opportunity to get to know potential applicants. As demonstrated by our webinar, social media has great potential as a means to provide resources, mentorship, and knowledge to students interested in ophthalmology. Additionally, social media and webinars may be utilized by stakeholders to increase exposure of all medical students to ophthalmology to further expand interest in and recruitment to the field among all students.

\section{Delivery and Conclusion}

At the end of our webinar, we administered a link to the recorded live session for anyone that could not attend but wished to view the session. ${ }^{9}$ Though we do not have access to the total number of views, we assume it has been widely disseminated. ${ }^{5}$ We also shared a .pdf document with key insights and information for those in attendance.

We felt that we could not adequately capture the passion of students that attended, and therefore we leave below various quotes from attendees. None of these quotes were solicited, rather they represent raw feedback of attendees.

\section{Direct Messages from the Audience}

"I always thought ophthalmology wasn't for me because I assumed my school would hold me back. Obviously that's not the case and you helped me see that. I wanted to share that you and your team provided me with insight that helped me consider all possibilities! So thank you" - DO student from Arizona without home program.

"The best webinar I have ever attended! I learned so much about the process of applying to ophthalmology, and got to hear from program directors. I was greatly inspired by the stories of residents who matched into their dream profession against all odds. I now feel inspired to give my $110 \%$." - Vincent Ngo, OD, OMSI TouroCOM- Middletown, NY

"I learned so much and left feeling incredibly inspired! Anyone applying ophthalmology should listen and learn!" - Dr. Andrea Tooley, Ophthalmic Plastic and Reconstructive Surgery Fellow at Columbia University.

"Glad I could be a part of it. Great meeting with great advice, I think the PDs also learned a lot hearing from the applicant's side." - Dr. Andrew Hendershot, Ohio State University Department of Ophthalmology.

"I'm so excited to be pursuing a field with so many physicians passionate about medical student mentorship!" - Victoria Ly, medical student from the University of Arkansas for Medical Sciences

"You have no idea how far the message has spread, all the way to Nepal. This webinar is probably the only resource of its kind for aspirants beyond boundaries: A light at the end of the tunnel." - Araniko Pandey, medical student, Palpa Lions Lacoul Eye Hospital, Palpa, Nepal

"Excellent Webinar. Another example of unity as our greatest asset in the challenging upcoming cycle." - Divy Mehra, DO student from Nova Southeastern University College of Osteopathic Medicine in Florida.

Conflict of Interest

None declared.

\section{References}

1 SF Match Residency and Fellowship Matching Services. 2020 OphthalmologyResidency Match Summary Report. Available at: https://sfmatch.org/PDFFilesDisplay/Ophthalmology_Residency_Stats_2020.pdf. Accessed May 22, 2020

2 Nallasamy S, Uhler T, Nallasamy N, Tapino PJ, Volpe NJ. Ophthalmology resident selection: current trends in selection criteria and improving the process. Ophthalmology 2010;117(05): 1041-1047

3 Loh AR, Joseph D, Keenan JD, Lietman TM, Naseri A. Predictors of matching in an ophthalmology residency program. Ophthalmology 2013;120(04):865-870

4 Schmuter G, Tooley AA, Chen RWS, et al. Social media in ophthalmology: the education and professional potential for medical students. J Acad Ophthalmol 2020;12:e41-e45

5 Tsui E, Rao RC, Carey AR, Feng MT, Provencher LM. Using social media to disseminate ophthalmic information during the \#COVID19 pandemic. Ophthalmology 2020 (e-pub ahead of print). Doi: 10.1016/j.ophtha.2020.05.048

6 Abugo U, Tooley A, Law J. Need a mentor? Want a mentee? [Internet]. https://www.surveymonkey.com/survey-closed/? sm=lgzrB_2BzeQOGo1ElxrhbPE_2BvPSRq9E9wkNL2gaoOQZKuDoGhxaH1kOUx9TEeYp91_2BF_2FDK9PZKsh_2B1v1qc9pVs_2FIBGfoLM1xrb_2F1RoRM439KU_3D. Accessed May 22, 2020

7 Shah M, Knoch D, Waxman E. The state of ophthalmology medical student education in the United States and Canada, 2012 through 2013. Ophthalmology 2014;121(06):1160-1163

8 Moxon NR, Goyal A, Giaconi JA, et al. The state of ophthalmology medical student education in the United States: an update. Ophthalmology 2020:S0161-6420(20)30425-5

9 Ahmed H, Felsted D, Song S, et al Matching in 2021 Amidst COVID19 [Webinar]. https://transcripts.gotomeeting.com/\#/s/ edaaf2595bf1fd87bf0c6ff46a3c04faa2754efd4a118eba067a2400aa4d62af. Accessed May 25, 2020

10 Association of American Medical Colleges. AAMC Medical School Members. Available at: https://members.aamc.org/eweb/DynamicPage.aspx? webcode=AAMCOrgSearchResult\&orgtype=Medical \%20School\#. Accessed May 22, 2020

11 American Association of Colleges of Osteopathic Medicine. US Colleges of Osteopathic Medicine. Available at: https://www. aacom.org/become-a-doctor/u-s-colleges-of-osteopathic-medicine. Accessed May 22, 2020 
e204 Editorial

12 Accreditation Council for Graduate Medical Education. Ophthalmology Programs Academic Year 2019-2020 United States. Available at: https://apps.acgme.org/ads/Public/Reports/ReportRun. Accessed May 22, 2020
13 Association of University Professors of Ophthalmology. Member Institutions. Available at: https://aupo.org/member-institutions. Accessed May 22, 2020 